\title{
Trombosis de senos venosos encefálicos como posible primera manifestación de enfermedad de Behçet: Caso clínico
}

\author{
JUAN CARLOS LIZARAZO-BARRERA ${ }^{1}$, SERGIO JACOBELLI ${ }^{1}$, \\ PATRICIO MELLADO ${ }^{2}$, SERGIO GONZÁLEZ ${ }^{3}$, LORETO MASSARDO ${ }^{1}$
}

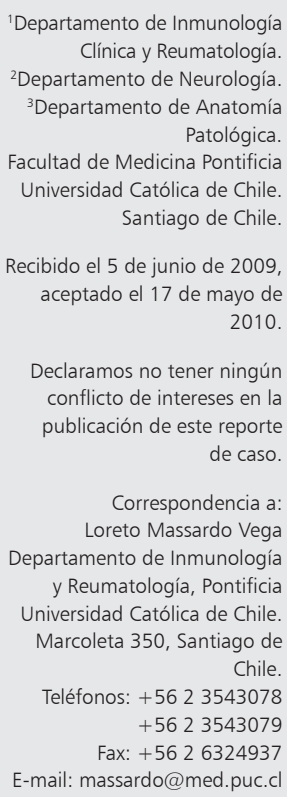

\section{Extensive cerebral vein thrombosis as first manifestation of Behçet's disease. Report of one case}

Behçet's disease (BD) is a systemic inflammatory vascular disease with several clinical manifestations and geographical differences in disease expression. In Middle Eastern countries it is one of the most common causes of cerebral venous thrombosis. We report a 29-year-old female admitted for acute headache and vomiting. A magnetic resonance image showed a large thrombosis of sagital and transverse sinuses. She developed oral and genital ulcers a week later. Ophthalmologic examination revealed left anterior uveitis and ipsilateral papilledema. Multiple studies ruled out a hypercoagulability syndrome. The patient used oral contraceptives. Anticoagulant therapy was prescribed. A biopsy of a genital ulcer demonstrated diffuse lymphocytic infiltration with vasculitis. After treatment with topical and systemic corticoids, her condition improved. Venous sinus thrombosis followed by oral and genital ulcers is an unusual presentation of Behçet's disease.

(Rev Med Chile 2010; 138: 746-751).

Key words: Behçet syndrome; Intracraneal thrombosis; Oral manifestations.
L a trombosis de senos venosos encefálicos (TSV) es un diagnóstico difícil y muchas veces elusivo pues las formas de presentación son síntomas neurológicos inespecíficos (náuseas, cefalea, vómitos, convulsiones). El diagnóstico se debe establecer mediante imágenes radiológicas como resonancia magnética $(\mathrm{RM})$ o tomografía contrastada de encéfalo (TC). Las causas de predisposición son numerosas (Tabla 1), sin embargo, hasta en $12,5 \%$ no se logra establecer y el $8,3 \%$ fallece ${ }^{1}$. Se presenta el caso de una mujer joven con TSV que consistió en la primera manifestación de una enfermedad de Behçet (EB).

\section{Caso clínico}

Paciente chilena, de 29 años de edad que consultó en el Servicio de Urgencia del Hospital Clínico de la Pontificia Universidad Católica de Chile en el mes de marzo de 2008 por cefalea global de 7 días que no mejoraba con analgésicos comunes y en las últimas 24 horas vómitos profusos no alimentarios por lo que ingresó al Servicio de Intermedio Neurológico. En el examen general era normal, no presentaba ulceraciones de mucosas y en la piel no tenía lesiones. Al examen neurológico había una rigidez de nuca leve y en el fondo de ojo 
Trombosis de senos venosos y enfermedad de Behçet - J. C. Lizarazo-Barrera et al

Tabla 1. Factores de riesgo en 624 enfermos del estudio internacional de trombosis venosa encefálica*1

\begin{tabular}{|lc|}
\hline Factores de riesgo para trombosis venosa encefálica & $\%$ \\
\hline Causa no identificada & 12,5 \\
\hline Trombofilia: Genética o adquirida incluido el síndrome antifosfolípido & 34,1 \\
\hline Postparto & 13,8 \\
\hline Infección: Cerebral, oído, nariz, cuello, cara & 12,3 \\
\hline Enfermedad Hematológica: Policitemia, trombocitemia, anemia & 12 \\
\hline Medicamentos: Anticonceptivos hormonales, citotóxicos, esteroides & 7,5 \\
\hline Embarazo & 6,3 \\
\hline Malignidad: Cerebral, extracraneal, hematológica, otras & 7,4 \\
\hline Anormalidades en el Sistema Nervioso Central: Fístula dural, anomalía venosa, malformación arteriovenosa & 4,6 \\
\hline Otras como deshidratación, enfermedad tiroidea, cardiopatía & 4,3 \\
\hline Precipitantes Mecánicos: Punción lumbar, trauma encefálico, oclusión catéter yugular, neurocirugía & 4,5 \\
\hline Vasculitis: Enf. Behçet, lupus, artritis reumatoide, tromboangiitis obliterante & 3 \\
\hline Cirugía & 2,7 \\
\hline Otras enfermedades sistémicas: Hipotiroidismo & 2,4 \\
\hline Otras enfermedades inflamatorias sistémicas: Sarcoidosis, enfermedad inflamatoria intestinal & 1,8 \\
\hline
\end{tabular}

*Modificado de 1.

edema de papila. El estudio con TC y RM de encéfalo demostró trombosis extensa de senos venosos asociada con signos de hipertensión intracraneana (Figura 1), por lo que se inició heparina intravenosa (IV). La paciente tenía historia de migraña sin aura desde los 15 años de edad con 6 episodios al año, no era fumadora y usaba anticonceptivos orales (ACO) por los últimos 4 años. Sin otros antecedentes mórbidos; sin embarazos y sin historia personal ni familiar de enfermedad reumatológica, la trombosis se atribuyó en ese momento al uso de ACO. El estudio para hipercoagulabilidad y para síndrome antifosfolípidos fue negativo y los exámenes generales fueron normales.

Una semana después del ingreso continuaba con cefalea pese a la analgesia utilizada. En la piel desarrolló un eritema con micropápulas, no pruriginoso, en la región peri umbilical, zona anterior y cara interna de los muslos. Además, por primera vez presentó múltiples úlceras orales muy dolorosas y refirió molestias genitales que se debían a la presencia de 5 úlceras genitales en labios mayores. Evaluada por ginecología se realizó la biopsia de una úlcera de la región de la vulva (Figura 2).

Las úlceras se trataron con corticoesteroide tópico (clobetazol 0,05\%) y lidocaína 3\% más colchicina $0,5 \mathrm{mg}$ oral cada 12 horas con una mejoría paulatina. Al día 11 del ingreso refirió visión borrosa del ojo izquierdo con ojo rojo correspondiente a una uveítis anterior que recibió

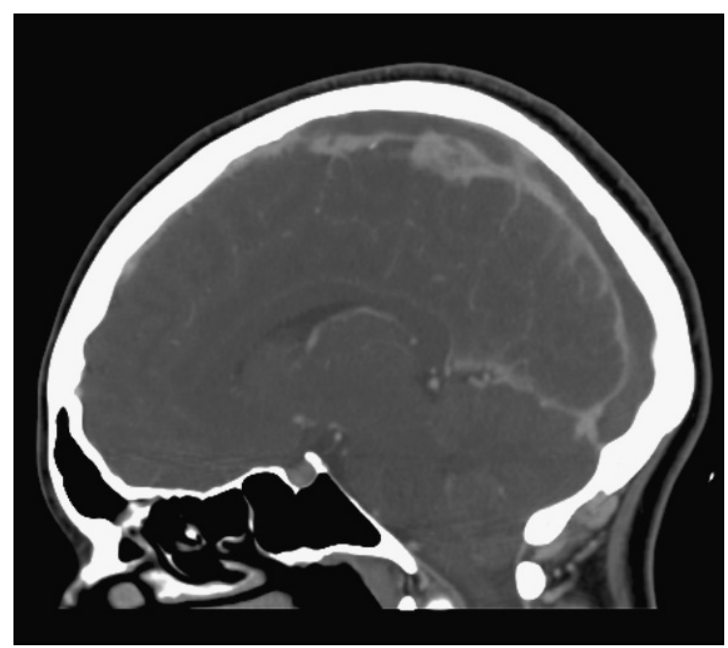

Figura 1. Reconstrucción sagital de tomografía computarizada de encéfalo con contraste que muestra ausencia de contraste en seno longitudinal superior, signo de un trombo extenso. 
corticoesteroides tópicos. Con el diagnóstico de EB se trató con metilprednisolona IV $250 \mathrm{mg}$ y continuó con prednisona $40 \mathrm{mg}$ día con completa resolución de la cefalea en horas. La RM al día 19 del ingreso mostró una recanalización parcial de los senos venosos.

Evolucionó en forma satisfactoria con resolución de las úlceras orales y mejoría de las lesiones genitales sin cicatrices. No se repitió la cefalea ni hubo defectos de visión atribuibles a la EB. Se agregó azatioprina $50 \mathrm{mg}$ día que no toleró por diarrea; como tenía niveles bajos de la enzima tiopurina metiltransferasa se suspendió definitivamente. Tres semanas después del ingreso se dio de alta con disminución progresiva de los corticoesteroides. Mantuvo anticoagulación durante 6 meses y 16 meses después del inicio de la enfermedad se encuentra en terapia sin corticoesteroides, con micofenolato mofetil 1 gr día y aspirina de 100 $\mathrm{mg}$, libre de síntomas.

\section{Discusión}

La TSV como modo de presentación de EB es infrecuente. En un estudio realizado en 820 pacientes con EB examinados entre los años 1974 y 2006 en Francia, la TSV se observó en 64 pacientes y en 14 de ellos fue su primera manifestación ${ }^{2}$. Hay dos estudios de pacientes chilenos con $\mathrm{EB}^{3}$ que reúnen un total 10 enfermos, siendo este el primer caso reportado en el país de presentación neurológica de la EB como cefalea intensa en mujer joven secundaria a trombosis de los senos

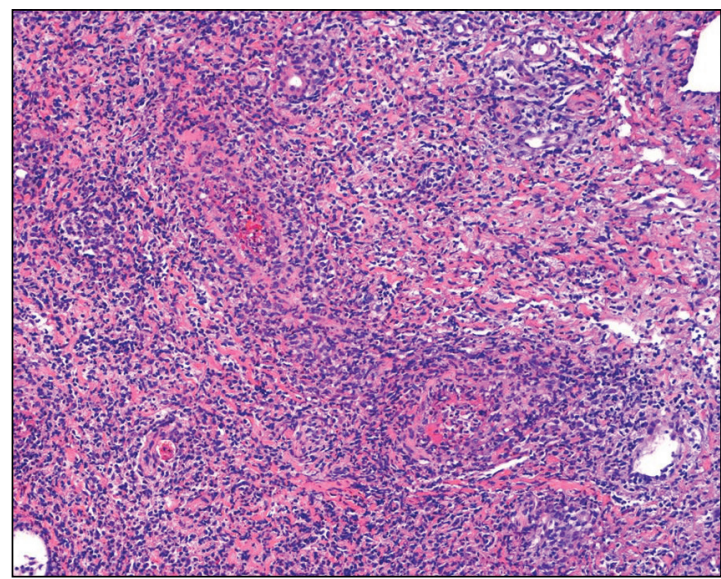

Figura 2. Biopsia de úlcera de mucosa de la vulva. Infiltrado inflamatorio difuso extenso compuesto de linfocitos, plasmocitos, abundantes granulocitos neutrófilos y focos de vasculitis con necrosis fibrinoide y microtrombos. HE, 100X

venosos encefálicos. Este reporte contribuye a la controversia sobre la inclusión del compromiso de grandes vasos en la clasificación diagnóstica "International Study Group (ISG) Diagnostic Criteria” para $\mathrm{EB}^{4}$ (Ver criterios diagnósticos en Tabla 2), como criterio adicional o en reemplazo de uno faltante, ya que puede ser observado antes que las úlceras orales y evolucionar sin necesariamente cumplir criterios ISG. Se postula que el reconocer esta situación clínica permitiría el diagnóstico temprano de EB lo que eventualmente afectaría favorablemente el pronóstico ${ }^{5,6}$.

El diagnóstico de EB lo hicimos por juicio clínico ya que la enferma presentó por primera

\section{Tabla 2. Criterios diagnósticos del International Study Group para la Enfermedad de Behçet ${ }^{4}$}

\begin{tabular}{ll|}
\hline Criterio & Características \\
Úlceras orales recurrentes & Por lo menos 3 episodios en un año de aftas o úlceras observadas por el médico o el paciente \\
$\begin{array}{l}\text { Más dos de las siguientes: } \\
\text { Úlceras } \\
\text { Genitales } \\
\text { Recurrentes }\end{array}$ & Úlceras o cicatrices observadas por el médico o por el paciente \\
\hline Lesiones oculares & Uveítis anterior o posterior o vasculitis retiniana observada por el oftalmólogo \\
\hline Lesiones de piel & $\begin{array}{l}\text { Eritema nodoso observado por el paciente o por el médico } \\
\text { Eritema papulopustuloso o pseudofoliculitis de tipo acneiforme observado por el médico en } \\
\text { pacientes post adolescentes que no reciben corticoesteroides }\end{array}$ \\
\hline Patergia & Evaluada de 24 a 48 horas por el médico \\
\hline
\end{tabular}


vez úlceras en mucosa oral y genital y uveítis sin cumplir el criterio principal obligatorio ISG para $\mathrm{EB}^{4}$ que es la recurrencia de las úlceras orales definidas como al menos tres episodios en un año y sí tenía dos criterios menores (uveítis y foliculitis). La sensibilidad y especificidad del juicio clínico se han establecido del orden de 1,00 y de 0,85 y para los criterios clasificatorios ISG se han estimado del orden de 0,95 y de 1,00, respectivamente ${ }^{7}$.

La EB es una entidad inflamatoria considerada como un tipo de vasculitis sistémica crónica de causa desconocida, con múltiples manifestaciones que se presenta más frecuentemente en menores de 30 años de edad ${ }^{8}$. La prevalencia es mayor en los países de la "ruta de la seda": Turquía, Japón, Corea, China, Irán y Arabia Saudita. En estos grupos étnicos hay una asociación genética con el antígeno de histocompatibilidad de clase I HLA-B51 y afecta más a hombres que a mujeres. En cambio, en Europa y Estados Unidos la prevalencia es menor, no se asocia con subtipos de HLA y afecta igualmente ambos sexos ${ }^{9,10}$. El diagnóstico de EB no es fácil, en un estudio hecho en Turquía el lapso promedio fue 6,6 años entre la primera manifestación y el diagnóstico de EB, estas dificultades podrían ser mayores en otras regiones en que las manifestaciones típicas son diferentes y la enfermedad menos frecuente ${ }^{5}$.

En este caso apoya el diagnóstico el que la paciente es HLA B51 positivo, tradicionalmente descrito en pacientes de origen oriental y asociado con el compromiso ocular ${ }^{6,10}$, sin embargo, la frecuencia de HLA B5 que incluye las especificidades B51 y B52 es 19\% en población chilena, en un estudio que comprendió 1.297 donantes o receptores de órganos ${ }^{11}$.

Las lesiones cutáneas son comunes en la EB entre $41 \%$ y $97 \%$ de los estudios, y para Schirmer y col${ }^{5}$ estarían sobre representadas en los criterios ISG. La paciente presentó foliculitis antes del uso de corticoesteroides y no tenía historia de patergia: se hizo observación de los sitios de punción venosa y catéteres y no se encontró alteración dérmica como pápulas, pústulas o abscesos estériles y la prueba fue negativa. La patergia es una reacción cutánea excesiva al trauma y varía durante el curso de la enfermedad. Si bien es un prueba relativamente específica de EB su sensibilidad es baja fuera de la "ruta de la seda"6. Aunque la histopatología no forma parte de los criterios diagnósticos y no es patognomónica, la biopsia de la úlcera genital fue compatible con EB: vasculitis con infiltración neutrofílica y necrosis fibrinoide (Figura 2).

El tratamiento de la EB se hace de acuerdo con los síntomas y territorios comprometidos, principalmente se han utilizado corticoesteroides tópicos y sistémicos, inmunosupresores y fármacos biológicos anti factor de necrosis tumoral (FNT) alfa ${ }^{12-14}$. Para el compromiso de mucosas, especialmente en genitales con lesiones que, si son de mayor tamaño, pueden dejar cicatrices ${ }^{15}$, se recomienda iniciar terapia con agentes como lidocaína combinada con corticoesteroides tópicos. Si esto es ineficaz se propone en forma escalonada usar colchicina, corticoesteroides sistémicos en bajas dosis, azatioprina o metotrexate y, en casos refractarios, infliximab o etanercept $\mathrm{t}^{16,17}$.

El tratamiento de primera elección en la uveítis anterior es la terapia tópica y para la uveítis posterior la ciclosporina o la azatioprina asociada con corticorticoesteroides sistémicos o, más recientemente, infliximab que ha mostrado eficacia en relación a reducción del número de episodios de uveítis, disminución de las dosis de esteroides y mejoría del pronóstico visual de los pacientes ${ }^{13,18}$. Hay pocos estudios de tratamiento controlados y aleatorios en EB. En estos estudios la colchicina, la ciclosporina y la azatioprina han demostrado superioridad respecto a placebo para el tratamiento tanto de las úlceras como de la uveítis ${ }^{14}$.

El compromiso de los grandes vasos es una las manifestaciones más complejas de la EB. En los países del medio oriente la EB es una de las principales causas de $\mathrm{TSV}^{19}$. La trombosis puede afectar a venas de las extremidades, la vena cava, las venas supra-hepáticas, los senos venosos encefáli$\cos$ y a casi cualquier territorio venoso o arterial. La terapia de la trombosis venosa incluye uso de inmunosupresores y anticoagulación ${ }^{2}$. Para aneurismas de las grandes arterias se recomienda el uso de citotóxicos como ciclofosfamida, combinado con dosis altas de corticoesteroides ${ }^{20}$.

En el caso de la paciente decidimos mantener anticoagulación por 6 meses y hacer inmunosupresión por al menos un año, dada la gravedad de los síntomas, de modo de prevenir la recaída. En la EB los inmunosupresores de elección son azatioprina o ciclosporina; sin embargo, la enferma tiene contraindicación para la primera pues es portadora de un déficit hereditario parcial de la enzima tiopurina metiltransferasa y la ciclosporina no fue aceptada al mencionarle los 
efectos colaterales. Se decidió probar respuesta a micofenolato de mofetil que es un inhibidor potente, selectivo, no competitivo y reversible de la inosinmonofosfato-deshidrogenasa que inhibe especialmente linfocitos $\mathrm{T}$ y $\mathrm{B}$, aunque no parece ser tan efectivo en comparación con azatioprina y ciclosporina ${ }^{21}$.

En esta paciente la TSV puede ser entendida como una suma de factores de riesgo. Tomaba anticonceptivos orales y aunque llevaba 4 años usándolos sin presentar ninguna trombosis tiene un proceso inflamatorio sistémico como es la EB, la que probablemente hizo que se desarrollara la trombosis. Se ha estimado el riesgo de presentar episodios trombóticos a nivel encefálico en el orden de 2 a 6 veces mayor comparado con las que no toman anticonceptivo ${ }^{22,23}$. En la actualidad la paciente no usa anticonceptivos hormonales.

La debilidad de este trabajo es la falta de certeza en el diagnóstico de EB de acuerdo con los criterios ISG, sin embargo, las múltiples manifestaciones de la enfermedad en un mismo sujeto pueden estar separadas por años y los criterios ISG no reemplazan al juicio clínico en casos individuales. Por otro lado, una falla en diagnosticar un paciente con EB pudiera afectar críticamente el pronóstico, en particular aquellos casos con manifestaciones vasculares de EB que no completan los criterios ISG dado que pudiera conducir a más complicaciones vasculares ${ }^{5}$. Así, se ha propuesto que la presencia de compromiso de grandes vasos o compromiso agudo de trombosis de senos venosos encefálicos o infarto agudo del sistema nervioso central en presencia de aftas sugiere fuertemente el diagnóstico en pacientes del hemisferio occidental ${ }^{6}$.

Agradecimientos: Al Dr. Jaime Pereira, hematólogo de la Pontificia Universidad Católica de Chile, por sus aportes en la hipótesis patogénica de la trombosis venosa de seno venoso encefálico. A los radiólogos del Hospital Clínico de la Pontificia Universidad Católica de Chile, Drs. Isidro Huete Lira, Rodrigo Parra Rojas y Juan Pablo Cruz Quiroga por la interpretación de las imágenes.

\section{Referencias}

1. Ferro JM, Canhao P, Stam J, Bousser MG, Barinagarrementeria F. Prognosis of cerebral vein and dural sinus thrombosis: results of the International Study on Cerebral Vein and Dural Sinus Thrombosis (ISCVT). Stroke
2004; 35: 664-70.

2. Saadoun D, Wechsler B, Resche-Rigon M, Trad S, Le Thi Huong D, Sbai A, et al. Cerebral venous thrombosis in Behcet's disease. Arthritis Rheum 2009; 61: 518-26.

3. Merino G, Varas G, Díaz G, Gutiérrez M, Massardo L, Pacheco D, et al. [Effectiveness of infliximab in patients with Behcet syndrome and severe uveoretinitis. Report of five cases]. Rev Med Chile 2006; 134: 875-82, López de Maturana D, Amaro P, Balestrini C, Segovia L. [Clinical manifestation in 5 cases of Behcet's disease]. Rev Med Chile 2002; 130: 551-6.

4. Criteria for diagnosis of Behcet's disease. International Study Group for Behcet's Disease. Lancet 1990; 335: 1078-80.

5. Schirmer M, Calamia Kt, O'Duffy JD. Is there a place for large vessel disease in the diagnostic criteria of Behcet's disease? J Rheumatol 1999; 26: 2511-2.

6. Calamia KT, Salvarani C. Vasculitides. E. Miscellaneous Vasculitis, in Primer on the Reumatic Diseases, J. H. Klippel, Editor-Editors. 2008, Springer: New York. p. 435-43.

7. Ferraz MB, Walter SD, Heymann R, Atra E. Sensitivity and specificity of different diagnostic criteria for Behcet's disease according to the latent class approach. Br J Rheumatol 1995; 34: 932-5.

8. Sakane T, Takeno M, Suzuki N, Inaba G. Behcet's disease. N Engl J Med 1999; 341: 1284-91, Yurdakul S, Hamuryudan V, Fresko I, Yazici H. Behcet's syndrome, in Rheumatology, M. Hochberg, A. Silman, J. Smolen, M. Weinblatt and M. Weisman, Editor^Editors. 2008, Mosby Elsevier. p. 1561-65.

9. Zouboulis CC, Kotter I, Djawari D, Kirch W, Kohl PK, Ochsendorf FR, et al. Epidemiological features of Adamantiades-Behcet's disease in Germany and in Europe. Yonsei Med J 1997; 38: 411-22.

10. Zouboulis CC. Epidemiology of Adamantiades-Behcet's disease. Ann Med Interne (Paris) 1999; 150: 488-98.

11. Droguett MA, Beltrán R, Ardiles R, Raddatz N, Labrana $\mathrm{C}$, Arenas A, et al. Ethnic differences in HLA antigens in Chilean donors and recipients: data from the National Renal Transplantation Program. Transplant Proc 2008; 40: 3247-50.

12. Hamuryudan V, Ozyazgan Y, Hizli N, Mat C, Yurdakul S, Tuzun Y, et al. Azathioprine in Behcet's syndrome: effects on long-term prognosis. Arthritis Rheum 1997; 40: 769-74.

13. Sfikakis PP, Markomichelakis N, Alpsoy E, Assaad-Khalil S, Bodaghi B, Gul A, et al. Anti-TNF therapy in the management of Behcet's disease-review and basis for recommendations. Rheumatology (Oxford) 2007; 46: 736-41. 
14. Yazici H, Fresko I, Yurdakul S. Behcet's syndrome: disease manifestations, management, and advances in treatment. Nat Clin Pract Rheumatol 2007; 3: 148-55.

15. Mat MC, Goksugur N, Engin B, Yurdakul S, Yazici H. The frequency of scarring after genital ulcers in Behcet's syndrome: a prospective study. Int J Dermatol 2006; 45: 554-6.

16. Melikoglu M, Fresko I, Mat C, Ozyazgan Y, Gogus F, Yurdakul S, et al. Short-term trial of etanercept in Behcet's disease: a double blind, placebo controlled study. J Rheumatol 2005; 32: 98-105.

17. Lin P, Liang G. Behcet disease: recommendation for clinical management of mucocutaneous lesions. J Clin Rheumatol 2006; 12: 282-6.

18. Kotter I, Zierhut M, Eckstein AK, Vonthein R, Ness T, Gunaydin I, et al. Human recombinant interferon alfa-2a for the treatment of Behcet's disease with sight threatening posterior or panuveitis. Br J Ophthalmol 2003; 87: 423-31.
19. Yesilot N, Bahar S, Yilmazer S, Mutlu M, Kurtuncu M, Tuncay R, et al. Cerebral venous thrombosis in Behcet's disease compared to those associated with other etiologies. J Neurol 2009; 256: 1134-42.

20. Calamia KT, Schirmer M, Melikoglu M. Major vessel involvement in Behcet disease. Curr Opin Rheumatol 2005; 17: 1-8.

21. Adler YD, Mansmann U, Zouboulis CC. Mycophenolate mofetil is ineffective in the treatment of mucocutaneous Adamantiades-Behcet's disease. Dermatology 2001; 203: 322-4.

22. Kemmeren JM, Tanis BC, Van Den Bosch MA, Bollen EL, Helmerhorst FM, Van Der Graa FY, et al. Risk of Arterial Thrombosis in Relation to Oral Contraceptives (RATIO) study: oral contraceptives and the risk of ischemic stroke. Stroke 2002; 33: 1202-8.

23. Dentali F, Crowther M, Ageno W. Thrombophilic abnormalities, oral contraceptives, and risk of cerebral vein thrombosis: a meta-analysis. Blood 2006; 107: 2766-73. 